\title{
Conditional purity and quantum correlation measures in two qubit mixed states
}

\author{
L. Rebón ${ }^{1,2}$, R. Rossignoli ${ }^{1,3}$, J.J.M. Varga ${ }^{4}$, N. Gigena ${ }^{1}$, N. \\ Canosa $^{1}$, C. Iemmi ${ }^{4}$, S. Ledesma ${ }^{4}$ \\ ${ }^{1}$ Instituto de Física de La Plata, Departamento de Física, CONICET, Universidad \\ Nacional de La Plata, C.C. 67, 1900 La Plata, Argentina. ${ }^{2}$ Institut für Laserphysik, \\ Universität Hamburg, Luruper Chaussee 149, 22761 Hamburg, Germany. ${ }^{3}$ CICPBA, \\ Argentina. ${ }^{4}$ Departamento de Física, FCEyN, Universidad de Buenos \\ Aires-CONICET, Buenos Aires 1428, Argentina. \\ E-mail: lrebon@PHYSnet.uni-hamburg.de
}

\begin{abstract}
We analyze and show experimental results of the conditional purity, the quantum discord and other related measures of quantum correlation in mixed twoqubit states constructed from a pair of photons in identical polarization states. The considered states are relevant for the description of spin pair states in interacting spin chains in a transverse magnetic field. We derive clean analytical expressions for the conditional local purity and other correlation measures obtained as a result of a remote local projective measurement, which are fully verified by the experimental results. A simple exact expression for the quantum discord of these states in terms of the maximum conditional purity is also derived.

PACS numbers: 03.67.-a, 03.65.Ud, 03.65.Ta,42.50.Ex
\end{abstract}




\section{Introduction}

Quantum correlations are at the heart of quantum information theory, constituting one of the key features that distinguishes quantum from classical composite systems. They are recognized as the essential resource that enables several quantum information processing schemes [1], such as quantum teleportation [2] and quantum algorithms exhibiting an exponential speed-up over their classical counterparts [3, 4]. Although quantum entanglement [5, 6] is the strongest type of quantum correlations, it does not encompass all non-classical correlations that can be exhibited by mixed states of composite systems. For such states, other measures of quantum-like correlations have been proposed [7], starting with quantum discord [8, 9, 10, 11, 12, 13], which was then followed by other related measures [7, 14, 15, 16]. The presence of discord-like correlations has been shown to be important in various quantum information processes [7], including quantum state merging [17], quantum state discrimination [18], quantum cryptography [19], quantum metrology [20] and quantum protocols [21]. Accordingly, several studies and verifications of these correlations in distinct contexts have been made [7, 22, 23, 24, 25, 26]. Furthermore, a quantum resource theory based on quantum coherence has recently been developed [27, 28] and some quantifiers of this resource have been directly related to measures of quantum discord [29, 30].

Quantum discord for a bipartite system can be defined [8] as the minimum difference between two distinct quantum generalizations of the classical conditional von Neumann entropy, one being the standard formal extension of the classical expression while the other one, introduced in [8, involves a local measurement on one of the constituents. The latter generalization measures the average conditional mixedness of the unmeasured component $(A)$ after the local measurement on the other component $(B)$ and is always a positive quantity, which is smaller or at least never larger than the original marginal entropy $S(A)$. Moreover, its minimum over all local measurements at $B$ is exactly the entanglement of formation between $A$ and a third system $C$ purifying the whole system [12. This fundamental relation has enabled, in particular, the connection with quantum state merging, a quantum information task where in a pure tripartite system $A B C, A$ transfers her state to $C$ through classical communication and shared entanglement. The minimal entanglement consumed in such process is given by the quantum discord between $A$ and $B$ (with measurements at $B$ ). The measurement dependent conditional entropy has been recently extended to more general entropic forms [31], where a similar relation with the corresponding generalized entanglement of formation holds. In particular, this generalization allows the use of simple forms like the linear entropy, which can be more easily evaluated and enables a clean analytical solution

of the associated optimization problem (i.e., that of selecting the local measurement which minimizes the conditional entropy) in general qudit-qubit states [31]. Moreover, such entropy is directly related to the purity, an experimentally accessible quantity whose determination does not require a full state tomography [32, 33], and which in the case of a qubit is formally related to the classical degree of polarization [34]. 
The aim of this work is to analyze and experimentally obtain the conditional purity, the quantum discord and other related measures of discord-like correlations [14, 16] in a particular class of mixed states which can be faithfully simulated through photonic quantum systems and linear optics [35, 36]. These states, which are mixtures of nonorthogonal aligned states, arise naturally in different contexts, in particular as reduced pair states in the exact ground state of spin 1/2 chains with anisotropic $X Y$ or $X Y Z$ couplings, in the immediate vicinity of the factorizing magnetic field [37, 38, 39]. In these chains they can also provide a basic description of reduced pair states in meanfield symmetry-breaking phases $\left(B<B_{c}\right)[22$.

For such states, described in section 2.1, we first derive in 2.2 2.3 simple analytical expressions for the conditional reduced state and its purity after a local measurement on one of the qubits, including the average conditional purity and its maximum among all possible local projective measurements. We then derive in sec. 2.4 a simple closed analytical expression for the quantum discord of these states in terms of the maximum conditional purity. Note that the computation of the quantum discord for general states is difficult due the associated minimization, having recently been shown to be an NP-complete problem [40]. Additionally, in 2.5 we present expressions for the global post-measurement purity and its minimizing measurement, which allows to evaluate the associated information deficit and the geometric discord. These quantities are also analyzed and compared. We have then experimentally tested these theoretical results using polarization-encoded photonic qubits that arise from a source emitting a pair of photons in the same polarization state, which enables to reproduce the mixed two qubit states. A description of the experimental setup used to prepare the desired state and perform the local measurements is given at the beginning of section 3.1 , with $[3.2$ devoted to present the experimental results and their comparison with the theoretical predictions. Conclusions are finally given in 4 .

\section{Theory}

\subsection{Initial state}

We consider the symmetric two qubit mixed state

$$
\rho_{A B}=p|\theta \theta\rangle\langle\theta \theta|+q|-\theta-\theta\rangle\langle-\theta-\theta|,
$$

where $|\theta \theta\rangle\langle\theta \theta|\equiv| \theta\rangle\langle\theta|\otimes| \theta\rangle\langle\theta|$, with

$$
| \pm \theta\rangle=\cos \frac{\theta}{2}|0\rangle \pm \sin \frac{\theta}{2}|1\rangle,
$$

pure single qubit states forming angles $\pm \theta$ with the $z$ axis on the Bloch sphere, and $p \in[0,1], q=1-p$ are the probabilities of preparing both qubits in the states $|\theta\rangle$ and $|-\theta\rangle$ respectively. Any rank 2 mixture of the form $\rho_{A B}=p|\Omega \Omega\rangle\left\langle\Omega \Omega|+q| \Omega^{\prime} \Omega^{\prime}\right\rangle\left\langle\Omega^{\prime} \Omega^{\prime}\right|$, with $|\Omega\rangle=\cos \frac{\theta}{2}|0\rangle+e^{i \phi} \sin \frac{\theta}{2}|1\rangle$ a general qubit state, can be rewritten at once in the form (11) by choosing a new $z$ axis in the Bloch sphere halfway between the directions $\Omega=(\theta, \phi)$ and $\Omega^{\prime}=\left(\theta^{\prime}, \phi^{\prime}\right)$ (and the $x$ axis in the plane determined by them). Moreover, 
any mixture $\rho_{A B}=p\left|\Omega_{1} \Omega_{2}\right\rangle\left\langle\Omega_{1} \Omega_{2}|+q| \Omega_{1}^{\prime} \Omega_{2}^{\prime}\right\rangle\left\langle\Omega_{1}^{\prime} \Omega_{2}^{\prime}\right|$, where the angle between $\Omega_{2}^{\prime}$ and $\Omega_{2}$ is identical with that between $\Omega_{1}^{\prime}$ and $\Omega_{1}$, can be also brought to the form (1) by applying local rotations on one of the qubits that shift $\Omega_{2}$ to $\Omega_{1}$ and $\Omega_{2}^{\prime}$ to $\Omega_{1}^{\prime}$. These rotations will not affect correlation measures.

Mixed states of the form (11) can arise in different contexts. For instance, they emerge naturally as reduced two-spin states in the ground state of ferromagnetic-type spin 1/2 arrays with anisotropic $X Y$ or $X Y Z$ couplings in the immediate vicinity of the transverse factorizing magnetic field [22, 38, 39], where the exact ground state becomes two-fold degenerate, being an arbitrary linear combination of uniform completely separable states, i.e.,

$$
|G S\rangle=\alpha|\theta \theta \ldots\rangle+\beta|-\theta-\theta \ldots\rangle,
$$

where $\theta$ is determined by the coupling anisotropy [38] (assumed constant for all coupled pairs). The state (3) leads to the reduced two-spin state (1) with $p=\left|\alpha^{2}\right|$, $q=\left|\beta^{2}\right|$ for any pair $i \neq j$, after tracing out the remaining qubits and neglecting the complementary overlap $\langle-\theta \mid \theta\rangle^{n-2}=\cos ^{n-2} \theta$, which decreases exponentially with increasing $n$ if $|\cos \theta|<1$. And in the mean field approximation, a reduced state of the form (11) with $p=q=1 / 2$ naturally arises in the whole parity breaking phase after parity symmetry restoration [22, 38, 39], becoming exact at the factorizing point.

The states (11) can also be generated using a source emitting a pair of photons in the same polarization state, e.g. by spontaneous parametric down-conversion (SPDC) produced in nonlinear crystal cut for type I phase matching [41, 42] and linear optics, such that

$$
| \pm \theta\rangle=\cos \frac{\theta}{2}|V\rangle \pm \sin \frac{\theta}{2}|H\rangle
$$

are linearly polarized states at angles $\pm \theta / 2$ with the vertical direction. Here $|V\rangle \equiv$ $|0\rangle,|H\rangle \equiv|1\rangle$ denote the orthogonal linearly polarized states in the vertical and horizontal directions respectively.

The purity of the state (1i) is given by

$$
P_{A B}=\operatorname{Tr} \rho_{A B}^{2}=1-2 p q\left(1-\cos ^{4} \theta\right),
$$

and is an increasing function of the overlap $|\langle-\theta \mid \theta\rangle|=|\cos \theta|$. Since $\rho_{A B}$ is a rank 2 state, the purity (5) completely determines its two non-zero eigenvalues,

$$
\lambda_{A B}^{ \pm}=\frac{1}{2}\left[1 \pm \sqrt{2 P_{A B}-1}\right]
$$

and hence, the value of any entropy

$$
S_{f}\left(\rho_{A B}\right)=\operatorname{Tr} f\left(\rho_{A B}\right)=f\left(\lambda_{A B}^{+}\right)+f\left(\lambda_{A B}^{-}\right),
$$

where $f$ is a concave function satisfying $f(0)=f(1)=0$ [43, 44]. In particular, the von Neumann entropy $S(\rho)$ corresponds to $f(\rho)=-\rho \log _{2} \rho$, while the linear entropy $S_{2}(\rho)$ to $f(\rho)=-2 \rho(1-\rho)$, in which case

$$
S_{2}\left(\rho_{A B}\right)=2\left(1-\operatorname{Tr} \rho_{A B}^{2}\right)=2\left(1-P_{A B}\right) .
$$

All entropies (7) will be decreasing functions of $P_{A B}$, vanishing iff $P_{A B}=1$. 
The state (1) is separable, i.e., a convex mixture of product states [6]. Nonetheless, if $\theta \in(0, \pi / 2)$ and $p q \neq 0$, it is not classically correlated, i.e., it is not diagonal in a standard or conditional product basis, having entangled eigenstates. It will then lead to a finite quantum discord (sec. 2.4).

The reduced state of each of the qubits (or photons) is

$$
\begin{aligned}
\rho_{B} & =\operatorname{Tr}_{A} \rho_{A B}=p|\theta\rangle\langle\theta|+q|-\theta\rangle\langle-\theta| \\
& =\frac{1}{2}\left(\begin{array}{cc}
1+\cos \theta & (p-q) \sin \theta \\
(p-q) \sin \theta & 1-\cos \theta
\end{array}\right)
\end{aligned}
$$

where (10) is the representation in the standard basis $\{|V\rangle,|H\rangle\}$, and corresponds to a Bloch vector $\boldsymbol{r}_{B}=\operatorname{Tr} \rho_{B} \boldsymbol{\sigma}=((p-q) \sin \theta, 0, \cos \theta)$. The local purity $P_{A}=P_{B}$ is then

$$
P_{B}=\operatorname{Tr} \rho_{B}^{2}=1-2 p q \sin ^{2} \theta
$$

with the eigenvalues of $\rho_{B}$ given by $\lambda_{B}^{ \pm}=\left(1 \pm \sqrt{2 P_{B}-1}\right) / 2$. It is verified that $P_{B} \geq P_{A B}$, $\lambda_{B}^{+} \geq \lambda_{A B}^{+}$, in agreement with the general majorization properties [45] $\rho_{A B} \prec \rho_{B(A)}$ valid for separable mixed states $\rho_{A B}$ [46, 47].

\subsection{Conditional local state and purity after remote local measurement}

Let us consider now a projective polarization measurement on photon $B$, defined by the orthogonal projectors

$$
\Pi_{+}=|\phi\rangle\left\langle\phi\left|, \quad \Pi_{-}=\right| \phi+\pi\right\rangle\langle\phi+\pi|,
$$

where $|\phi\rangle=\cos \frac{\phi}{2}|V\rangle+\sin \frac{\phi}{2}|H\rangle,|\phi+\pi\rangle=-\sin \frac{\phi}{2}|V\rangle+\cos \frac{\phi}{2}|H\rangle$ and $\Pi_{+}+\Pi_{-}=1$. This means projecting onto linearly polarized states at angles $\phi / 2$ and $\phi / 2+\pi / 2$ respectively. The probability of obtaining result + or - is

$$
r_{ \pm}=\operatorname{Tr}\left(\rho_{A B} I_{A} \otimes \Pi_{ \pm}\right)=\frac{1}{2}[1 \pm p \cos (\phi-\theta) \pm q \cos (\phi+\theta)] .
$$

(Obviously, a result "+" for measurement angle $\phi$ is equivalent to a result "-" for measurement angle $\phi+\pi)$. As a function of $\phi, r_{ \pm}$is extremum for

$$
\tan \phi=(p-q) \tan \theta
$$

with $r_{+}$maximum for $\phi=0$ if $p=q$ and $\phi$ between 0 and $\theta$ if $p>q$ (Fig. 1).

After a measurement at $B$ with known result, the post-measurement state of the unmeasured photon will have again the form (91), but with modified probabilities $p_{ \pm}^{\prime}, q_{ \pm}^{\prime}$, which depend on both the measurement angle $\phi$ and measurement result \pm :

$$
\rho_{A / B_{ \pm}}=r_{ \pm}^{-1} \operatorname{Tr}_{B}\left(\rho_{A B} I_{A} \otimes \Pi_{ \pm}\right)=p_{ \pm}^{\prime}|\theta\rangle\left\langle\theta\left|+q_{ \pm}^{\prime}\right|-\theta\right\rangle\langle-\theta|,
$$

with $q_{ \pm}^{\prime}=1-p_{ \pm}^{\prime}$ and

$$
p_{ \pm}^{\prime}=p \frac{1 \pm \cos (\theta-\phi)}{2 r_{ \pm}}
$$

It is of course verified that $r_{+} \rho_{A / B_{+}}+r_{-} \rho_{A / B_{-}}=\rho_{A}$, i.e., that the post-measurement state at $A$ is unchanged if the result is unknown. It is also seen that if $\phi=0$ (or 

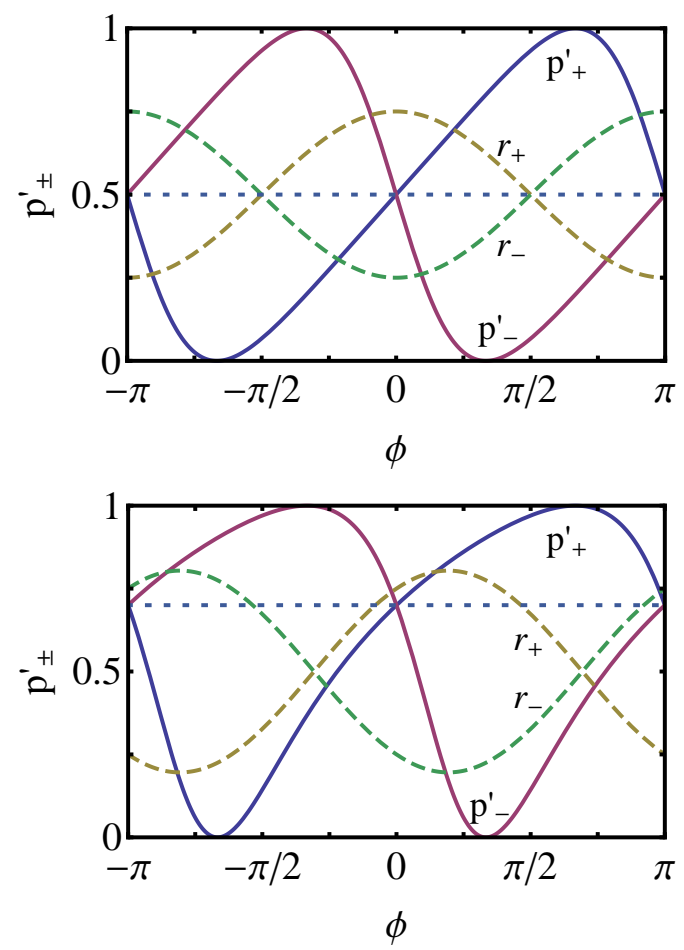

Figure 1. (Color online) The probabilities $p_{ \pm}^{\prime}$, Eq. (16), of the conditional reduced states (15) of $A$ after a measurement with result \pm at $B$ along angle $\phi$, for $\theta=\pi / 3$ and initial values $p=0.5$ (top) and $p=0.7$ (bottom), indicated by the horizontal dotted lines. It is seen that $p_{ \pm}^{\prime}$ cover all values between 0 and 1 , with $p_{+}^{\prime}=1$ at $\phi=\pi-\theta$ and 0 at $\phi=\theta-\pi$, while $p_{-}^{\prime}=0$ at $\phi=\theta$ and 1 at $\phi=-\theta$. The dashed lines depict the probabilities $r_{ \pm}$, Eq. (13), of obtaining result \pm at $B$.

$\phi=\pi), p_{ \pm}^{\prime}=p$, i.e., $\rho_{A / B_{ \pm}}=\rho_{A}$ irrespective of the values of $\theta, p$ and the result of the measurement. Such measurement then leaves the marginal state of the unmeasured photon (but not the whole $\rho_{A B}$ ) unchanged.

As seen in Fig. 1, the new probabilities $p_{ \pm}^{\prime}$ cover all possible values from 0 to 1 as the measurement angle $\phi$ is varied. For $p>q, p_{+}^{\prime}\left(p_{-}^{\prime}\right)$ stays above (below) the initial value $p$ for $\phi \geq 0$, and the opposite behavior takes place for $\phi \leq 0$. The purities of the states (15) are given by

$$
P_{A / B_{ \pm}}=1-2 p_{ \pm}^{\prime} q_{ \pm}^{\prime} \sin ^{2} \theta=1-2 p q \frac{[1 \pm \cos (\theta-\phi)][1 \pm \cos (\theta+\phi)]}{4 r_{ \pm}^{2}} \sin ^{2} \theta
$$

and can then be larger or smaller than the original purity (11), satisfying

$$
1-\frac{1}{2} \sin ^{2} \theta \leq P_{A / B_{ \pm}} \leq 1
$$

For $P_{A / B_{-}}$, the upper limit is always reached if $\phi= \pm \theta$, as in this case a - result implies with certainty a pure post-measurement state $|\mp \theta\rangle$ in $A$, i.e., $p_{-}^{\prime}=0$ or 1 , as verified in Fig. 1. Such result has probability $r_{-}=q \sin ^{2} \theta\left(p \sin ^{2} \theta\right)$ if $\phi=\theta(-\theta)$. The same occurs for $P_{A / B_{+}}$if $\phi=\mp(\pi-\theta)$. On the other hand, the lower limit in (18) corresponds 
to $p_{ \pm}^{\prime}=1 / 2$ and can be reached for angles $\phi$ satisfying

$$
\tan \phi=\frac{(p-q) \sin \theta}{\cos \theta \pm 2 \sqrt{p q}}
$$

in which case $p_{-}^{\prime}=1 / 2$ for the roots of $(19) \in[0, \pi]$ while $p_{+}^{\prime}=1 / 2$ for those $\in[-\pi, 0]$, as seen in Fig. 1, Hence by suitable measurements and results at $B$ it is always possible to have the conditional post-measurement state at A pure or also "equilibrated", i.e., with equal weights of both states of the mixture.

\subsection{Average conditional purity}

The average conditional purity of $A$ after the previous measurement at $B$ is given by

$$
\begin{aligned}
P_{A / B_{\phi}} & =r_{+} P_{A / B_{+}}+r_{-} P_{A / B_{-}} \\
& =1-2 p q \gamma \sin ^{2} \theta,
\end{aligned}
$$

where

$$
\gamma=\frac{r_{+} p_{+}^{\prime} q_{+}^{\prime}+r_{-} p_{-}^{\prime} q_{-}^{\prime}}{p q}=\frac{p \sin ^{2}(\theta-\phi)+q \sin ^{2}(\theta+\phi)}{1-[p \cos (\theta-\phi)+q \cos (\theta+\phi)]^{2}} \leq 1 .
$$

Hence, in contrast with $P_{A / B_{ \pm}}, P_{A / B_{\phi}}$ is never lower than the original purity:

$$
P_{A / B_{\phi}} \geq P_{A}
$$

in agreement with the general results of [31]. Eq. (20) is in fact linearly related to the measurement dependent $S_{2}$ conditional entropy [31], which becomes here

$$
\begin{aligned}
S_{2}\left(A / B_{\phi}\right) & =r_{+} S_{2}\left(\rho_{A / B_{+}}\right)+r_{-} S_{2}\left(\rho_{A / B_{-}}\right) \\
& =2\left(1-P_{A / B_{\phi}}\right)=4 p q \gamma \sin ^{2} \theta,
\end{aligned}
$$

and is never greater than the original local entropy $S_{2}\left(\rho_{A}\right)=4 p q \sin ^{2} \theta$.

The difference $P_{A / B_{\phi}}-P_{A}=2 p q(1-\gamma) \sin ^{2} \theta$ is the average conditional purity gain at $A$ due to the local measurement at $B$, and depends on the measurement angle $\phi$. While it always vanishes for $\phi=0$, where $\gamma=1$, it is otherwise positive. Its maximum is attained for

$$
\tan \phi=\frac{\tan \theta}{p-q}
$$

in which case $\gamma=\cos ^{2} \theta$, leading to

$$
P_{A / B} \equiv \operatorname{Max}_{\phi} P_{A / B_{\phi}}=1-2 p q \sin ^{2} \theta \cos ^{2} \theta \text {. }
$$

Moreover, at this point $p_{+}^{\prime}=q_{-}^{\prime}$ and hence,

$$
P_{A / B_{+}}=P_{A / B_{-}}=P_{A / B}
$$

so that the maximum average gain is attained at an angle where the post-measurement local purity (but not the local state) is independent of the result of the measurement (see also Fig. 4 in sec. 3.2). The maximum average purity gain is thus $2 p q \sin ^{4} \theta$. 
The maximum average conditional purity (25) has a deep significance. The associated minimum $S_{2}$ conditional entropy

$$
S_{2}(A / B)=\operatorname{Min}_{\phi} S_{2}\left(A / B_{\phi}\right)=2\left(1-P_{A / B}\right)=p q \sin ^{2} 2 \theta
$$

represents the squared concurrence [48] between $A$ and a third system $C$ purifying the whole system $A B C$ [31, 12, 17]. $C$ can be here chosen as a single qubit due to the rank 2 of $\rho_{A B}$. As a consequence (see next subsection), the maximum average conditional purity (25) will also determine the quantum discord of the state (1), enabling a simple analytical expression for the latter. The behavior of $P_{A / B}$ as a function of the "aperture" angle $\theta$ of the state (1) is depicted on Fig. 2, where it is seen that it reaches its maximum 1 just for $\theta=0$ or $\pi / 2$, i.e., when the state (1) is either a product state $(\theta=0)$ or a classically correlated state $(\theta=\pi / 2)$, i.e., a state of zero discord in both cases.

The maximizing $\phi$ determined by (24) differs from $\pm \theta$ if $\theta>0, p q>0$, as seen in the bottom panel of Fig. 2. It satisfies $\phi \geq \theta$ if $p \geq q$, with $\phi \approx \theta+(1-p) \sin 2 \theta$ for $p$ close to 1 and $\phi \approx \frac{\pi}{2}-2\left(p-\frac{1}{2}\right) / \tan \theta$ for $p$ above and close to $1 / 2$. Hence, for $p=q$ it becomes independent of $\theta$, preferring always a measurement along the $x$ axis in the Bloch sphere (i.e., projecting onto linearly polarized states at angles $\pm \pi / 4$ ), as seen in Fig. 2.

The previous feature is in agreement with the general considerations of [31]. The measurement direction $\boldsymbol{k}$ in the Bloch sphere of $A$ maximizing the conditional purity of $B$ is essentially that of maximum correlation and satisfies the generalized eigenvalue equation [31] $C^{T} C \boldsymbol{k}=\lambda N_{B} \boldsymbol{k}$, with $\lambda$ the largest eigenvalue, where $C_{\mu \nu}=$ $\left\langle\sigma_{\mu}^{A} \otimes \sigma_{\nu}^{B}\right\rangle-\left\langle\sigma_{\mu}^{A}\right\rangle\left\langle\sigma_{\nu}^{B}\right\rangle$ is the correlation tensor of the system and $N_{B}=I_{3}-\boldsymbol{r}_{B} \boldsymbol{r}_{B}^{T}$, with $\boldsymbol{r}_{B}=\left\langle\boldsymbol{\sigma}^{B}\right\rangle$ the original Bloch vector of qubit $B$. Here $\left.\boldsymbol{r}_{A}=\boldsymbol{r}_{B}=((p-q) \sin \theta, 0, \cos \theta)\right)$ and $C_{\mu \nu}=\delta_{\mu \nu} \delta_{\mu x} 4 p q \sin ^{2} \theta$, so that correlations arise just along the $x$ direction. The previous eigenvalues equation then leads to a maximizing $\boldsymbol{k}$ in the $x z$ plane, i.e., $\boldsymbol{k}=(\sin \phi, 0, \cos \phi)$, with $\phi$ satisfying Eq. (24). And for $p=q, \boldsymbol{k}$ becomes parallel to the $x$ axis as $N_{B}$ becomes diagonal.

\subsection{Quantum Discord and its analytical evaluation}

As previously mentioned, the state (11) has a finite quantum discord for $\theta \in(0, \pi)$ and $p q \neq 0$. As a function of the measurement angle $\phi$, this quantity [ $[$, 9] can be evaluated as the minimum of the difference

$$
D\left(A / B_{\phi}\right)=S\left(A / B_{\phi}\right)-S(A / B),
$$

where $S(A / B)=S\left(\rho_{A B}\right)-S\left(\rho_{B}\right)=-\sum_{\nu= \pm}\left(\lambda_{A B}^{\nu} \log _{2} \lambda_{A B}^{\nu}-\lambda_{B}^{\nu} \log _{2} \lambda_{B}^{\nu}\right)$ is the standard von Neumann conditional entropy [43] and

$$
S\left(A / B_{\phi}\right)=r_{+} S\left(\rho_{A / B_{+}}\right)+r_{-} S\left(\rho_{A / B_{-}}\right),
$$

the measurement dependent von Neumann conditional entropy [8], determined by the conditional states (15) (analogous to Eq. (23)). Hence, all quantities can be evaluated in terms of the purities $P_{A B}, P_{A}$ and $P_{A / B_{ \pm}}$(Eqs. (15), (11) and (17) respectively). 

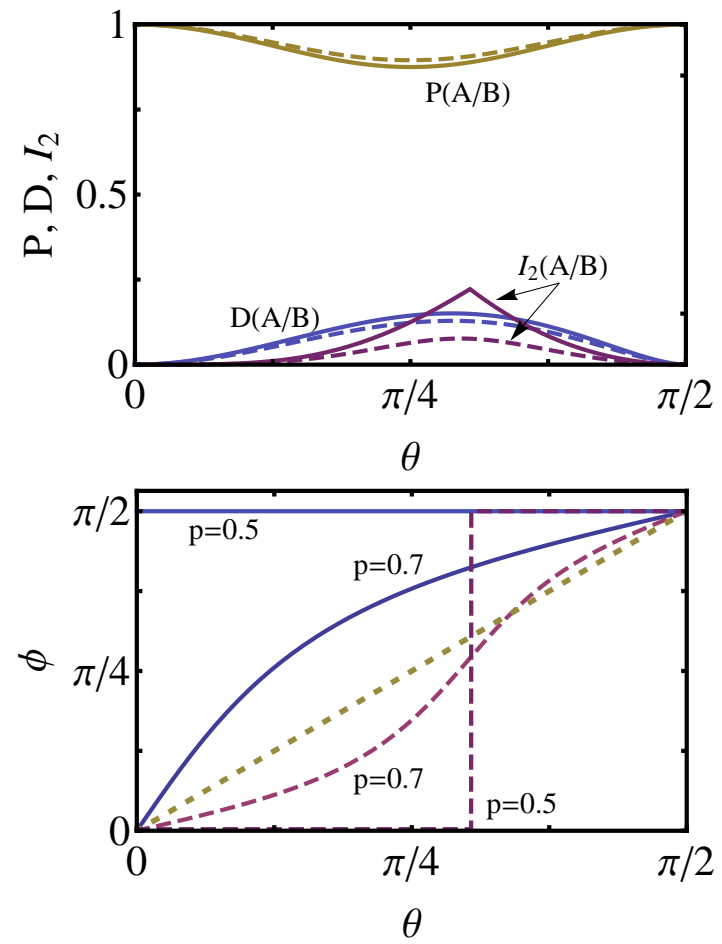

Figure 2. (Color online) Top: The maximum average conditional purity $P(A / B) \equiv$ $P_{A / B}$, Eq. (25), together with the quantum Discord, Eq. (31) and the minimum global purity difference, Eq. (36), as a function of the aperture angle $\theta$ of the state (11) for $p=0.5$ (solid lines) and $p=0.7$ (dashed lines). Bottom: The measurement angles which maximize the average conditional purity (20) (solid lines, Eq. (24) and the global post-measurement purity (33) (dashed lines, Eq. (37)), as a function of the aperture angle $\theta$ for $p=0.5$ and $p=0.7$. The dotted line depicts $\theta$ for reference. For $p=0.5$, the angle maximizing (33) undergoes a sharp $0 \rightarrow \pi / 2$ transition at $\theta=\arccos 1 / \sqrt{3}$, which originates the sharp peak in $I_{2}$ seen in the top panel and which becomes smoothed out for $p>1 / 2$. The angle minimizing the quantum discord (28) coincides here exactly with that maximizing the conditional purity (20) (see text).

The actual quantum discord $D(A / B)$ is the minimum over $\phi$ of Eq. (28) [8, 13]. The minimization should in principle be extended to general POVM measurements, but in the present case of a rank 2 state, it is sufficient to consider just projective measurements [7, 49], which can here be reduced to a measurement in the $x z$ plane. Furthermore, the minimum of (28) can be here evaluated analytically: The minimizing measurement angle $\phi$ is exactly that which maximizes the average conditional purity, determined by Eq. (24), and the ensuing minimum is a decreasing function of the maximum average conditional purity $P_{A / B}$, Eq. (25) (even though for general $\phi$, (28) is not a direct function of (20)). We obtain

$$
D(A / B)=\operatorname{Min}_{\phi} D\left(A / B_{\phi}\right)=-f_{+} \log _{2} f_{+}-f_{-} \log _{2} f_{-}-S(A / B)
$$

where

$$
f_{ \pm}=\frac{1 \pm \sqrt{2 P_{A / B}-1}}{2}
$$


Proof: According to the result of [12], the minimum of $S\left(A / B_{\phi}\right)$ is the entanglement of formation $E(A, C)$ between $A$ and a closing third system $C$ purifying the whole system, which can be chosen here as a single qubit. In such a case, $E(A, C)$ is determined by the concurrence [48] $C_{A C}$ between $A$ and $C$ through $E(A, C)=-\sum_{\nu= \pm} f_{\nu} \log f_{\nu}$, with

$f_{ \pm}=\left(1 \pm \sqrt{1-C_{A C}^{2}}\right) / 2$. But $C_{A C}^{2}$ is just the minimum $S_{2}$ conditional entropy (27) of $A$ given a measurement at $B$ [31], i.e. $C_{A B}^{2}=S_{2}(A / B)=2\left(1-P_{A / B}\right)$, which leads to Eqs. (30)-(31). We have also verified this result numerically.

For $\theta=0$ ( $\rho_{A B}$ product state) or $\theta=\pi / 2$ ( $\rho_{A B}$ classically correlated), $P_{A / B}=1$ and hence $D(A / B)=0$. Otherwise $P_{A / B}<1$ and $D(A / B)>0$, as appreciated in Fig. 2. Note, however, that as a function of $\theta, P_{A / B}$ is minimum at $\pi / 4$ (Eq. (25)), while $D(A / B)$ is maximum at a slightly higher aperture angle $\theta \approx 0.29 \pi$, due to the $\theta$-dependence of the term $S(A / B)$.

\subsection{Global post-measurement purity}

The average state of the whole system after the previous local measurement at $B$ is

$$
\rho_{A B}^{\prime}=r_{+} \rho_{A / B_{+}} \otimes \Pi_{+}+r_{-} \rho_{A / B_{-}} \otimes \Pi_{-} .
$$

Its purity $P_{A B}^{\prime}=\operatorname{Tr}\left(\rho_{A B}^{\prime}\right)^{2}$ is then given by

$$
\begin{aligned}
P_{A B}^{\prime} & =r_{+}^{2} P_{A / B_{+}}+r_{-}^{2} P_{A / B_{-}} \\
& \left.=\frac{1}{2}\{p \cos (\theta-\phi)+q \cos (\theta+\phi)]^{2}-2 p q \sin ^{2} \theta[1+\cos (\theta+\phi) \cos (\theta-\phi)]\right\} .
\end{aligned}
$$

In contrast with Eq. (22), this global post-measurement purity cannot be greater than the original global purity (5), in agreement with the general considerations of [16]:

$$
P_{A B}^{\prime} \leq P_{A B}
$$

with $P_{A B}^{\prime}<P_{A B}$ for $\theta \in(0, \pi / 2)$ and $p q>0$. The difference $P_{A B}-P_{A B}^{\prime}$ is just proportional to the $S_{2}$ information deficit [16],

$$
I_{2}\left(A, B_{\phi}\right)=S_{2}\left(\rho_{A B}^{\prime}\right)-S_{2}\left(\rho_{A B}\right)=2\left(P_{A B}-P_{A B}^{\prime}\right),
$$

which is always non-negative. Eq. (33) shows that it can also be evaluated just with the conditional purities $P_{A / B_{ \pm}}$, the initial purity $P_{A B}$ and the probabilities $r_{ \pm}$.

Its minimum

$$
I_{2}(A, B)=\operatorname{Min}_{\phi} I_{2}\left(A, B_{\phi}\right)
$$

which corresponds to maximum global post-measurement purity $P_{A B}^{\prime}$, is proportional to the geometric discord [14, 16, 26]. It will then be non-zero for $\theta \in(0, \pi / 2)$ and $p q \neq 0$, being maximum, like the quantum Discord, at an angle $\theta$ above $\pi / 4$, as appreciated in Fig. 2. A Renyi entropy based information deficit $I_{2}^{R}\left(A, B_{\phi}\right)=-\log P_{A B}^{\prime} / P_{A B}$ can also be directly obtained from $I_{2}$ [53.

The measurement angle $\phi$ maximizing $P_{A B}^{\prime}$ (and minimizing $\left.I_{2}\left(A, B_{\phi}\right)\right)$ satisfies

$$
\tan 2 \phi=\frac{(p-q) \sin 2 \theta}{p q+(1-p q) \cos 2 \theta} .
$$


It is not greater than that maximizing $P_{A / B_{\phi}}$ (Eq. (24) $)$ and can be larger or smaller than $\theta$, with $\phi \approx \theta-(1-p) \cos ^{2} \theta \sin 2 \theta$ for $p \rightarrow 1$. On the other hand, for $p \rightarrow 1 / 2$, $\phi \rightarrow \pi / 2$ just for $\cos \theta \leq 1 / \sqrt{3}$, i.e. $\theta>\theta_{c} \gtrsim 0.309 \pi$, with $\phi \rightarrow 0$ for $\cos \theta>1 / \sqrt{3}$. Hence, for $p=1 / 2$ a sharp transition from 0 to $\pi / 2$ in the maximizing measurement angle of $P_{A B}^{\prime}$, occurs at $\theta=\theta_{c}$ [16. Such sharp transition becomes smoothed out for $p>q$, as seen in Fig. 2,

Since at fixed $\theta$, the minimizing angle $\phi$ of (35) can differ from that minimizing the quantum discord, the behavior of (35) as a function of the measurement angle $\phi$ may become out of phase with that of the quantum discord, as will be appreciated in Fig. 5. In particular, for $p=q$ and $\theta<\theta_{c}$, (35) is minimum at $\phi=0$, where $P_{A / B_{\phi}}$ is minimum and hence $D\left(A / B_{\phi}\right)$ is maximum (as a function of $\phi$ ). This difference is

reflecting the distinct meaning of the optimizing angles of $P_{A / B_{\phi}}$ and $D\left(A / B_{\phi}\right)$ on one side, and $I_{2}\left(A, B_{\phi}\right)$ on the other side. While the former chooses essentially the local direction associated with maximum correlation, the latter represents the direction of a least disturbing local measurement [16], which produces the smallest global purity decrease. Accordingly, differences can be significant for small aperture angles $\theta$, where the latter will be closer to the $z$ axis, but will decrease as $\theta$ increases, as seen in the bottom panel of Fig. 2. For $p=q$ they vanish in fact for $\theta>\theta_{c}$.

Let us finally remark that the direction $\boldsymbol{k}$ in the Bloch sphere of $B$ of the measurement minimizing (35) satisfies the standard eigenvalue equation [14, 16] $\left(J^{T} J+\right.$ $\left.\boldsymbol{r}_{B} \boldsymbol{r}_{B}^{T}\right) \boldsymbol{k}=\lambda \boldsymbol{k}$, with $\lambda$ the maximum eigenvalue, where $J_{\mu \nu}=\left\langle\sigma_{\mu}^{A} \otimes \sigma_{\nu}^{B}\right\rangle=C_{\mu \nu}+$ $\left\langle\sigma_{\mu}^{A}\right\rangle\left\langle\sigma_{\nu}^{B}\right\rangle$. In the present situation $\boldsymbol{k}$ will lie in the $x z$ plane, i.e., $\boldsymbol{k}=(\sin \phi, 0, \cos \phi)$, with $\phi$ satisfying Eq. (37). Actually, the maximizing $\phi$ is the smallest positive root of (37), the other root corresponding to the angle minimizing $P_{A B}^{\prime}$ (lowest eigenvalue).

\section{Experimental verification}

\subsection{Experimental setup}

The experimental setup is depicted in Fig. 3. It can be divided in three stages. In the first part, used for state preparation, a LiIO3 nonlinear crystal cut for type-I phasematching, is pumped by an horizontally polarized $405 \mathrm{~nm}$ laser diode, that by means of spontaneous parametric down conversion (SPDC) produces pairs of twin photons with wavelength $\lambda=810 \mathrm{~nm}$ in the two-qubit polarization state $|V V\rangle$. A half-wave plate $\left(\mathrm{HWP}_{1}\right)$ is used to rotate the polarization of each photon to an arbitrary direction $\theta_{L}$ in the laboratory reference system, where $\theta_{L}=0$ corresponds to vertically polarized photons. This angle defines the state $|\theta\rangle$ in the Bloch sphere through the relation $\theta=2 \theta_{L}$. In order to generate the mixed two-qubit states described by Eq. (11), we followed the idea presented in [50] switching $\mathrm{HWP}_{1}$ between the two angles $\theta_{L} / 2$ and $-\theta_{L} / 2$ to obtain a mixture of the two desired polarizations with probabilities $p$ and $q$.

In the second part of the setup, a local projective polarization measurement is done in one of the subsystems. To this end, a linear polarizer $\left(\mathrm{P}_{1}\right)$ in the path $B$, set at an 
angle $\phi_{L}$ or $\phi_{L}+\pi / 2$ in the laboratory reference system, implements the action of the projector $\Pi_{+}$or $\Pi_{-}$of Eq. (12) on the single qubit state. After that, the light is collected by the detector $\mathrm{D}_{B}$. The detector is conformed by an iris that acts as spatial filter, and an interference filter centered at 810nm (10 nm bandwidth), followed by a lens that collects the light and focuses it in a multi mode optical fiber coupled to a photon counting module PerkinElmer SPCM-AQRH-13-FC. During the total time $T$ that $\mathrm{P}_{1}$ is set at the angle $\phi_{L}, \mathrm{D}_{B}$ measures the number of single counts $n_{+}$, and the same is done when $\mathrm{P}_{1}$ is set at the complementary angle $\phi_{L}+\pi / 2$ to register $n_{-}$. Then, the probability of measuring + or - along the direction $\phi\left(r_{ \pm}\right)$given in Eq. (13) is obtained as $n_{ \pm} /\left(n_{+}+n_{-}\right)$.

Finally, the third part of the set up is used to perform a complete single-qubit tomography on the subsystem $A$. An array of a quarter-wave plate (QWP), a half-wave plate $\left(\mathrm{HWP}_{2}\right)$, and a linear polarizer $\left(\mathrm{P}_{2}\right)$ in the path of the subsystem is used to project the polarization state onto the informational complete set of mutually unbiased basis [51, 52], before being detected by $\mathrm{D}_{A}$. This detector consists of the same components than $\mathrm{D}_{B}$, which was described above. The measurements are performed in coincidence, using the subsystem $B$ as a trigger. In this way it is possible to reconstruct the conditional density matrix $\rho_{A / B= \pm}$ of the reduced state of $A$ after obtaining the outcome \pm of the projective measurement along direction $\phi$ at $B$. Although these observables could be obtained by performing a conditional purity measurement, the used set up aditionally allows to verify that the post-measurement local conditional state was of the form (15).

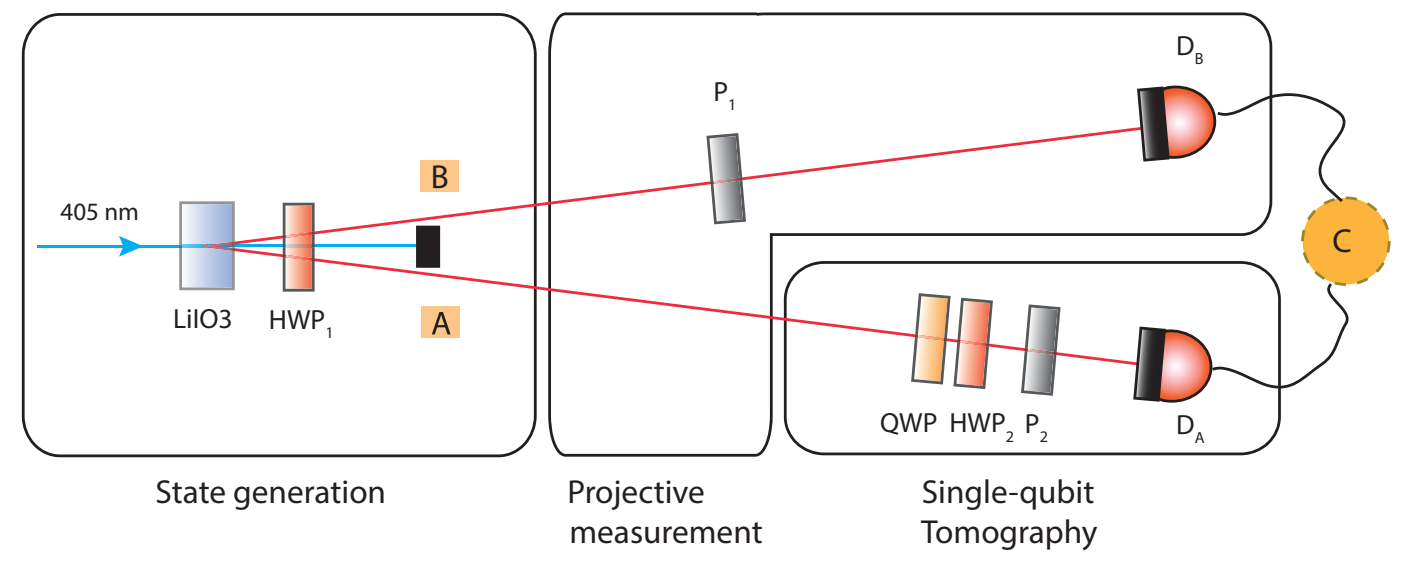

Figure 3. Experimental setup used to the preparation of a mixed polarization two-qubits state, and characterization of the single-qubit state in $A$ conditional to a projective measurement at $B$. QWP: quarter-wave plate; HWP: half-wave plate; P: linear polarizer; D: single photon detector.

\subsection{Results}

As mentioned above, the first section of the experimental setup generates a two-photon field in the polarization state given by Eq. (1). In order to validate the generation 

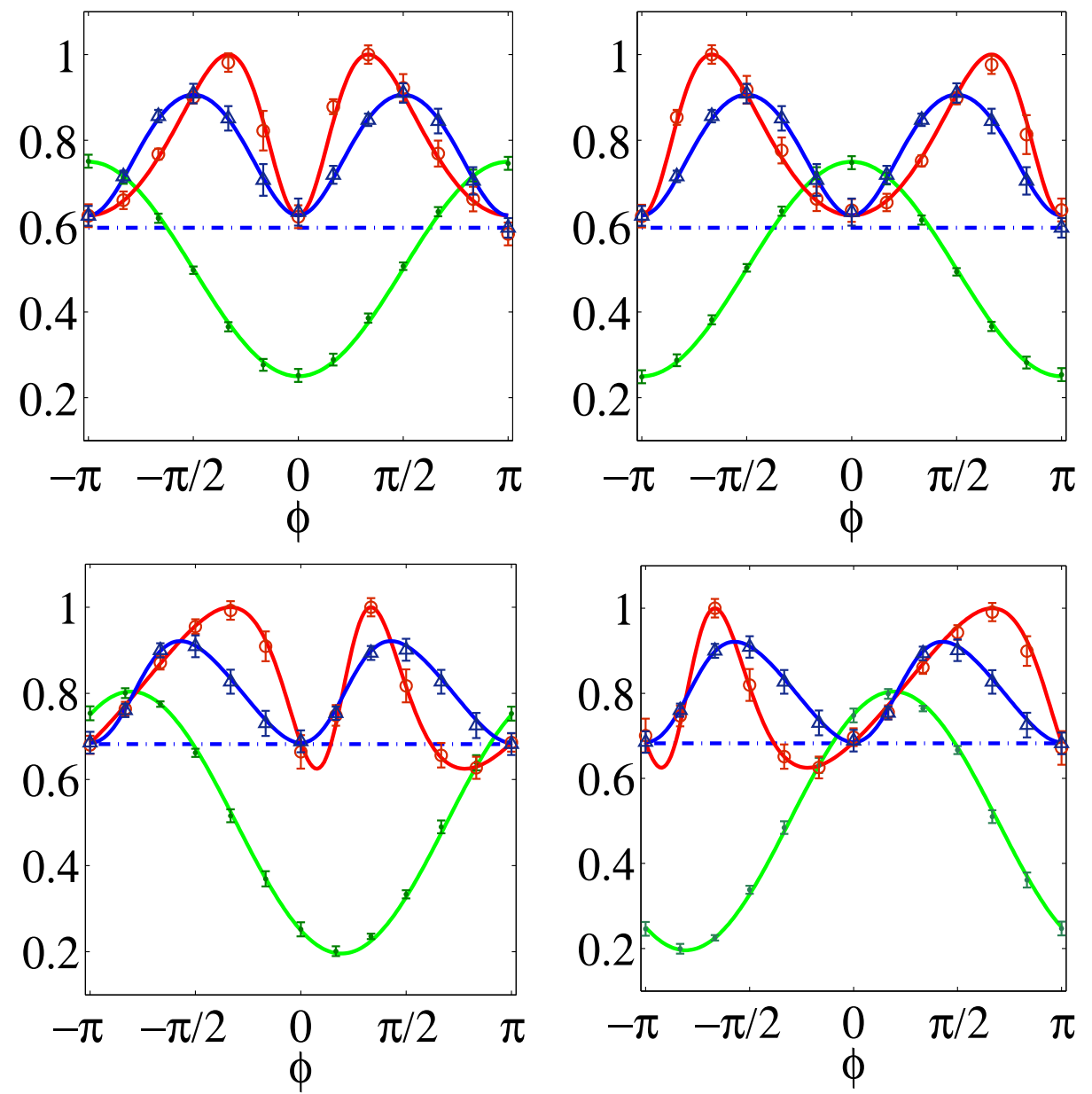

Figure 4. (Color online) Experimental results obtained from each projective measurement described by $\Pi_{ \pm}$. It shows the purity of the state $A$ after obtaining the result - (left panels) or + (right panels) at $B, P_{A / B_{ \pm}}$(Eq. (17)) (red circles), and the probability to obtain this result, $r_{ \pm}$(Eq. (13)) (green points). Additionally, each graphic shows the average conditional purity $P_{A / B_{\phi}}$, Eq. (20) (blue triangles). In all cases the initial state is a two-qubit state with $\theta=\pi / 3$. For the top panels $p=0.5$ and for the bottom panels $p=0.7$. Solid lines correspond to the theoretical values for these measures as a function of $\phi$. The dashed line indicates the measured value of the local purity $P_{A}$ in the initial state before conditional measurement.

process, a previous complete tomography [54] for different mixed two-qubit states was done. Afterwards, maximum likelihood technique (ML) was applied to obtain the best state estimation consistent with the requirements of a physical state [55]. We quantified the quality of the preparation process by means of the fidelity $F \equiv \operatorname{Tr}(\sqrt{\sqrt{\sigma} \rho \sqrt{\sigma}})$ between the density matrix of the state intended to be prepared, $\sigma$, and the density matrix of the state actually prepared and reconstructed by tomography, $\rho$. In all cases, fidelities $F>0.98$ for the initial state $\rho_{A B}$ were obtained. After this previous characterization we performed the projective measurements and the conditional onequbit tomography implemented in the second and third parts of the setup (Fig. 3). The 

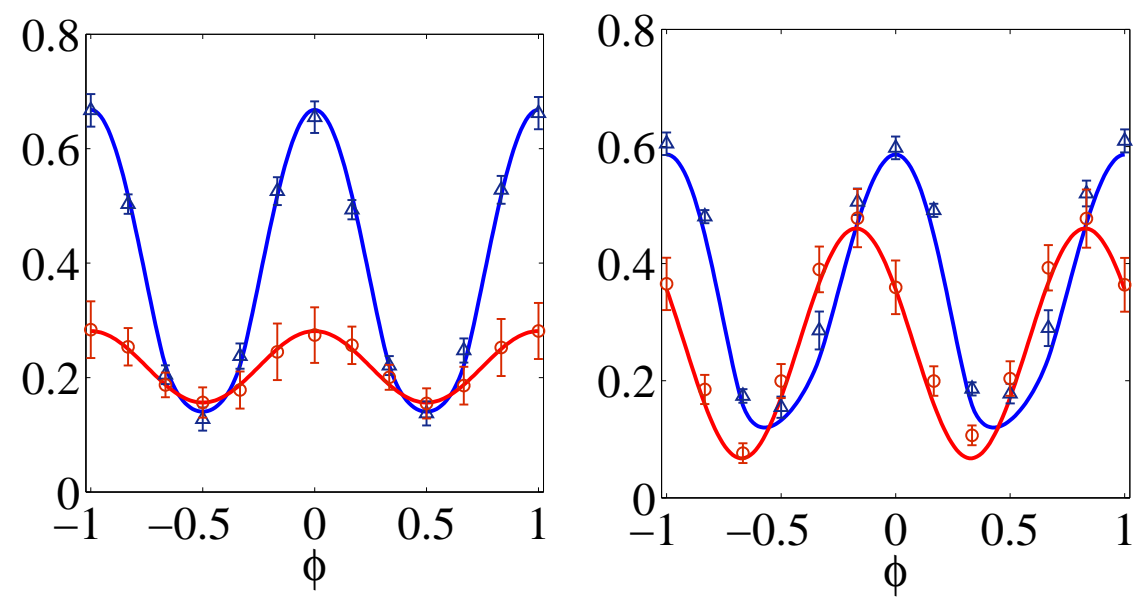

Figure 5. (Color online) Experimental results obtained from each projective measurement described by $\Pi_{ \pm}$. The minimum of the blue line (triangles) is the value of the quantum discord while the minimum of the red line (circles) is the value of geometric discord (minimum global purity difference). The initial state is a two qubit state corresponding to $\theta=\pi / 3$ with $p=0.5$ (left panel) and $p=0.7$ (right panel). Solid lines correspond to the theoretical values for these measures as a function of the measurement angle $\phi$.

post measurement state $\rho_{A / B_{ \pm}}$in Eq. (15) was obtained after applying the ML technique to the experimental results. The plots in Fig. 4 show the probabilities for the projective measurements $r_{ \pm}$and the conditional purities $\operatorname{Tr} \rho_{A / B_{ \pm}}^{2}$ for two different initial states together with the theoretical predictions given by Eqs. (13) and (17). Additionally, we plot the average conditional purity $P_{A / B_{\phi}}$ which was obtained from the experimental results as $r_{+} P_{A / B_{+}}+r_{-} P_{A / B_{-}}$and theoretically as $1-2 p q \gamma \sin ^{2} \theta$ (Eq. (20)). In Fig. 5 we plot the experimental and theoretical results for $D(A / B)$ and $I_{2}\left(A, B_{\phi}\right)$, whose minimum values as a function of the projective measurement $\Pi_{\phi}$ correspond to the quantum discord and the geometric discord, respectively. As follows from Sec. 2.4 and Sec. 2.5 both quantities can be evaluated from the purities $P_{A B}, P_{A}$, and $P_{A / B_{ \pm}}$. For this purpose, the density matrix of the reduced state $\rho_{A}$ was obtained in a similar way to $\rho_{A / B_{ \pm}}$but considering the single counts in $D_{A}$ without taking in account the results in $D_{B}$.

\section{Conclusions}

We have examined in full detail the quantum correlation properties of the two-parameter mixed states (11), characterized by an aperture angle $\theta$ and a probability or weight $p$. Such states arise naturally as reduced two-spin states of spin $1 / 2$ chains, either at the mean field approximation level or exactly in the vicinity of the factorizing field, and can be easily generated by photonic qubit states in a linear-optics architecture. We have derived simple exact analytical expressions for the conditional purity of one of the qubits after a local measurement on the other qubit, including its maximum average. These quantities allow one to also determine quantum correlation measures. 
In particular, we have derived a simple exact analytical expression for the quantum discord of the state, which unveils its direct connection with the previous maximum average conditional purity, valid for the present states. This result enables a straightforward experimental evaluation of the quantum discord of the state through a conditional single photon purity measurement. Such determination can assess, for instance, its potential for quantum protocols such as quantum state merging. The global post-measurement purity and the associated information deficit $I_{2}$ were as well analytically evaluated and compared with the previous measures. The experimental results showed a remarkable agreement with the theoretical predictions. The analysis indicates that the form of the reduced state of the unmeasured photon $A$ remains unchanged after a remote measurement on photon $B$, although selection of the measurement angle allows full control of the probabilities characterizing this reduced state, including pure and maximally mixed limits. At the same time, due to the non-

orthogonality of the states involved these probabilities do affect the eigenstates of the conditional reduced states, and any local measurement does affect the average postmeasurement global state. Possible applications to cryptography and metrology are currently under investigation.

\section{Acknowledgments}

The authors acknowledge support from CIC (RR) and CONICET(LR,JJMV,NG,NC,CI,SL) of Argentina. L.R. thanks the Institut für Gravitationsphysik and Leibniz Universität Hannover for kind hospitality. This work was supported by UBACyT 20020130100727BA, CONICET PIP 112201101-00902, CONICET PIP 11220080103047, and ANPCYT PICT 201002179 (Argentina).

\section{References}

[1] Nielsen M and Chuang I L 2000 Quantum Computation and Quantum Information (UK: Cambridge Univ. Press, Cambridge).

[2] Bennett C H et al 1993 Phys. Rev. Lett. 701895.

[3] Jozsa R and Linden N 2003 Proc. R. Soc. London Ser. A 459 2011; Vidal G 2003 Phys. Rev. Lett. 91147902.

[4] Raussendorf R and Briegel H J 2001 Phys. Rev. Lett. 86 5188; Raussendorf R, Browne D E and Briegel H J 2003 Phys. Rev. A 68, 022312.

[5] Schumacher S 1995 Phys. Rev. A 512738.

[6] Werner R F 1989 Phys. Rev. A 404277.

[7] Modi K et al 2012 Rev. Mod. Phys. 841655.

[8] Ollivier H and Zurek W H 2001 Phys. Rev. Lett. 88017901.

[9] Henderson L and Vedral V 2001 J. Phys. A 346899.

[10] Vedral V 2003 Phys. Rev. Lett. 90050401.

[11] Zurek W H 2003, Phys. Rev. A 67012320.

[12] Koashi M and Winter A 2004 Phys. Rev. A 69022309.

[13] Datta A, Shaji A, Caves C M 2008 Phys. Rev. Lett. 100050502.

[14] Dakić B, Vedral V and Brukner Č 2010 Phys. Rev. Lett. 105190502. 
[15] Streltsov A, Kampermann H and Bruß D Phys. Rev. Lett. 106, 160401 (2011); Piani M et al 2011 Phys. Rev. Lett. 106220403.

[16] Rossignoli R, Canosa N, Ciliberti L 2010 Phys. Rev. A 82 052342; 2011 Phys. Rev. A 84052329.

[17] Cavalcanti D et al 2011 Phys. Rev. A 83 032324; Madhok V and Datta A 2011 Phys. Rev. A 83 032323. Fanchini F F et al 2011 Phys. Rev. A 84012313.

[18] Roa L, Retamal J C and Alid-Vaccarezza M 2011 Phys. Rev. Lett. 107080401.

[19] Pirandola S 2014 Scientific Reports 46956.

[20] Girolami D, Tufarelli T and Adesso G 2013 Phys. Rev. Lett. 110240402.

[21] Chuan Bobby T K, Paterek T 2014 New J. Phys. 16093063.

[22] Ciliberti L, Rossignoli R, Canosa N 2010 Phys. Rev. A 82 042316; 2013 Phys. Rev. A 880211219.

[23] Wang C et al, 2011 J. Phys. B 44015503.

[24] Adhikari S, Banerjee S 2012 Phys. Rev. A 86062313.

[25] Hosseini S et al 2014 J. Phys. B 47025503.

[26] Qiang W, Zhang L and Zhang H 2015, J. Phys. B 48244503.

[27] Baumgratz T, Cramer M, Plenio M B 2014, Phys. Rev. Lett. 113140401.

[28] Streltsov A et al, 2015 Phys. Rev. Lett. 115020403.

[29] Yao Y, Xiao X, Ge L, Sun C P Phys. Rev. A 92022112.

[30] Matera J M, Egloff D, Killoran N, Plenio M B, 2016 Quantum Sci. Technol. 1 01LT01.

[31] Gigena N and Rossignoli R 2014 J. Phys. A 47 015302; 2014 Phys. Rev. A 90042318.

[32] Filip R 2002 Phys. Rev. A 65 062320; Nakazato H et al 2012 Phys. Rev. A 85 042316; Tanaka T et al 2013 Phys. Rev. A 87 012303; Bartkiewicz K, Lemr K and Miranowicz A 2013 Phys. Rev. A 88052104.

[33] Brun T A 2004 Quantum Information and Computation 4 401; Bovino F A et al 2005 Phys. Rev. Lett. 95 240407; Du J et al 2006 Phys. Rev. A 74 042319; Adamson R B A, Shalm L K and Steinberg A M 2007 Phys.Rev. A 75012104.

[34] Gamel O, James D F B 2012 Phys. Rev. A 86033830 (2012).

[35] Orieux A, Boutari J, Barbieri M, Paternostro M and Mataloni P 2014 Sci. Rep. 47184.

[36] Xiao-Song Ma et. al 2014 Sci. Rep. 43583.

[37] Kurmann J, Thomas H and Müller G 1982 Physica A 112235.

[38] Rossignoli R, Canosa N, Matera J M 2008 Phys. Rev. A 77 052322; 2009 Phys. Rev. A 80062325.

[39] Canosa N, Rossignoli R and Matera J M 2010 Phys. Rev. B 81054415.

[40] Huang Y 2014 N. J. Phys. 16033027.

[41] Burnham D C and Weinberg D L 1970 Phys. Rev. Lett. 2584.

[42] Mandel L and Wolf E 1995 Optical coherence and quantum optics (New York: Cambridge University Press).

[43] Wehrl H 1978 Rev. Mod. Phys. 50221.

[44] Canosa N and Rossignoli R 2002 Phys. Rev. Lett. 88170401.

[45] Bhatia R 1997 Matrix Analysis (New York: Springer-Verlag).

[46] Nielsen M A and Kempe J 2001 Phys. Rev. Lett. 865184.

[47] Rossignoli R and Canosa N 2003 Phys. Rev. A 67 042302; Rossignoli R and Canosa N 2002 Phys. Rev. A 66042306.

[48] Hill S and Wootters W K 1997 Phys. Rev. Lett. 78 5022; Wootters W K 1998 Phys. Rev. Lett. 802245.

[49] Galve F, Giorgi G L, Zambrini R 2011 Eur. Phys. J. 96 40005; Shi M, Yang W, Jiang F, Du J 2011 J. Phys. A 44415304.

[50] Amselem E and Bourennane M 2009 Nat. Phys. 5748.

[51] Ivanovic I D 1981 J. Phys. A 143241.

[52] Wootters W K and Fields B D 1989 Ann. Phys. 191363.

[53] Canosa N, Ciliberti L, Rossignoli R 2015 Entropy 171634.

[54] James D F V, Kwiat P G, Munro W J and White A G 2001 Phys. Rev. A 64052312.

[55] Fiurášek J 2001 Phys. Rev. A 64024102. 